\title{
Free Flap Reconstruction of Head and Neck Defects after Oncologic Ablation: One Surgeon's Outcomes in 42 Cases
}

\author{
Yun Sub Lim ${ }^{1}$, Jun Sik Kim ${ }^{1}$ Nam Gyun Kim ${ }^{1}$, Kyung Suk Lee ${ }^{1}$, Jae Hoon Choi ${ }^{1}$, \\ Sang Woo Park ${ }^{2}$ \\ ${ }^{I}$ Department of Plastic and Reconstructive Surgery, Institute of Health Science, Gyeongsang National University Hospital, Gyeongsang National \\ University School of Medicine, Jinju; ${ }^{2}$ Department of Plastic and Reconstructive Surgery, Changwon Samsung Medical Center, Sungkyunkwan \\ University School of Medicine, Changwon, Korea
}

Background Free flap surgery for head and neck defects has gained popularity as an advanced microvascular surgical technique. The aims of this study are first, to determine whether the known risk factors such as comorbidity, tobacco use, obesity, and radiation increase the complications of a free flap transfer, and second, to identify the incidence of complications in a radial forearm free flap and an anterolateral thigh perforator flap.

Methods We reviewed the medical records of patients with head and neck cancer who underwent reconstruction with free flap between May 1994 and May 2012 at our department of plastic and reconstructive surgery.

Results The patients included 36 men and 6 women, with a mean age of 59.38 years. The most common primary tumor site was the tongue (38\%). The most commonly used free flap was the radial forearm free flap (57\%), followed by the anterolateral thigh perforator free flap (22\%). There was no occurrence of free flap failure. In this study, risk factors of the patients did not increase the occurrence of complications. In addition, no statistically significant differences in complications were observed between the radial forearm free flap and anterolateral thigh perforator free flap.

Conclusions We could conclude that the risk factors of the patient did not increase the complications of a free flap transfer. Therefore, the risk factors of patients are no longer a negative factor for a free flap transfer.

Keywords Free tissue flaps / Postoperative complication / Risk factors

\author{
Correspondence: Jun Sik Kim \\ Department of Plastic and \\ Reconstructive Surgery, Institute of \\ Health Science, Gyeongsang National \\ University Hospital, Gyeongsang \\ National University School of \\ Medicine, 79 Gangnam-ro, \\ Jinju 660-702, Korea \\ Tel: +82-55-758-8647 \\ Fax: +82-55-758-6240 \\ E-mail: junskim@gshp.gsnu.ac.kr
}

No potential conflict of interest relevant to this article was reported.

\section{INTRODUCTION}

Reconstruction of the head and neck is challenging due to the variety of tissues whose structural deficiencies must be corrected [1-3]. This is because the defects include a variety of structures: skin, mucosa, soft tissue, and bone. In particular, the anatomy of the oral cavity is complicated, and each structure plays a specific role in speech, swallowing, and facial expression. In addition, defects in one specific functional unit can affect adjacent structures [1-3]. Before reconstruction, a comprehensive assessment 
of the defect is required. Disease status and tumor staging may also affect postoperative treatment and outcomes.

Reconstruction options for defects of the head and neck include primary closure, skin graft, local flap, pedicled flap, and vascularized free flap transfer [1-3]. In the 1970 to 1980s, the local flap or pedicled flap was performed for coverage of defects. However, although the pedicle flap was adequate for reconstruction, several limitations, such as revision surgery for a bulky flap, limited arc of rotation, and partial flap necrosis due to decreased blood flow of the flap distal portion, remained. In addition, reconstruction using a pedicled flap is impossible in cases involving an accompanying bone defect [3-5]. Recently, free flap surgery for surgical defects of the head and neck has gained popularity as an advanced microvascular surgical technique [2,3]. A literature review uncovered reports of successful performance of free flap transfer for oncological surgical defects of the head and neck. However, free flap failure remains a challenging problem [5]. The goals of this study are first, to determine whether the known risk factors such as comorbidity, tobacco use, obesity, and radiation increase the complications of free flap transfer and, second, to identify the incidence of complications in radial forearm free flap (RFFF) and anterolateral thigh perforator flap (ALTPF).

\section{METHODS}

We conducted a retrospective review of medical records of patients with head and neck cancer who underwent reconstruction with free flap transfer between May 1994 and May 2012 at our department of plastic and reconstructive surgery. A total of 42 patients were considered. All free flap transfer procedures were performed immediately after cancer ablation by a single senior surgeon. All of the medical records were reviewed retrospectively for patients' characteristics, including body mass index (BMI), comorbidity, history of smoking or alcohol use, tumor characteristics, preoperative radiotherapy, type of reconstruction surgery, and complications. Complications were classified as major complications and minor complications. The major complications

\section{Table 1. Patient characteristics}

\begin{tabular}{|lc|}
\hline Characteristic & Value \\
\hline Sex (male:female) & $36: 6$ \\
Mean age (yr) & 59.4 \\
Smoking & 30 \\
Alcohol & 28 \\
Diabetic mellitus & 3 \\
Hypertension & 6 \\
Preoperative radiotherapy & 2 \\
Mean operation time $(\mathrm{min})$ & 301 \\
Mean body mass index $\left(\mathrm{kg} / \mathrm{m}^{2}\right)$ & 23 \\
\hline
\end{tabular}

were defined as flap loss, arterial thrombosis, and venous thrombosis, and the minor complications were defined as flap dehiscence, leakage, and fistula. A statistical analysis was performed using a commercially available statistical software package, SPSS ver. 18.0 (SPSS Inc., Chicago, IL, USA). Fisher's exact test was used for the identification of factors associated with free flap complications and for an evaluation of the differences in the incidence of complications between RFFF and ALTPF. Statistical significance was defined as $\mathrm{P}<0.05$.

\section{RESULTS}

Between May 1994 and May 2012, 50 patients underwent reconstruction for defects of the head and neck. Among them, 42 patients underwent free flap transfer for surgical defects of the head and neck. All free flap transfer procedures were performed immediately after cancer ablation. These patients included 36 men and 6 women, with a mean age of 59.4 years. Three patients had diabetes, 6 patients had hypertension, and 30 patients had a history of tobacco use. The average amount of smoking was 31 pack years, and 28 patients had a history of alcohol use. The mean BMI was $23 \mathrm{~kg} / \mathrm{m}^{2}$. Two patients had a history of preoperative radiation therapy. The average operation time was 301 minutes (Table 1 ). The most common primary tumor site was the tongue (31.8\%), followed by the tonsils, and the mouth floor. The most common tumor diagnosis was squamous cell carcinoma (76\%) (Table 2). The most commonly used free flap transfer was RFFF (57\%), followed by ALTPF (22\%). The other free flap transfers were the fibular free flap, latissimus dorsi myocutaneous free flap, dorsalis pedis free flap, and jejunal free flap (Table 3). The most common recipient artery was the facial artery, in 36 cases, followed by the superior thyroid artery, in 6 cases. Complications occurred in 10 patients (24.8\%), major

\section{Table 2. Tumor profile}

\begin{tabular}{|lrlr|}
\hline Primary tumor site & \multicolumn{3}{c|}{ Tumor cell type } \\
\hline Tongue & 16 & Squamous cell carcinoma & 38 \\
Tonsil & 5 & Adenoid cystic carcinoma & 1 \\
Mouth floor & 4 & Papillary carcinoma & 1 \\
Hypopharynx & 3 & Lymphoepithelial carcinoma & 1 \\
Buccal mucosa & 3 & Spindle cell carcinoma & 1 \\
Pyriform sinus & 2 & & \\
Larynx & 2 & & \\
Gingiva & 2 & & \\
Subglottis & 1 & & \\
Soft palate & 1 & & \\
Mandible & 1 & \\
Lower lip & 1 & \\
Esophagus & 1 & & \\
Total & 42 & Total \\
\hline
\end{tabular}




\section{Table 3. Type of flap}

\begin{tabular}{|lc|}
\hline Type of flap & No. (\%) \\
\hline Radial forearm free flap & $24(57.1)$ \\
Anterior lateral thigh perforator flap & $8(19.0)$ \\
Fibular free flap & $6(14.3)$ \\
Latissimus dorsi myocutaneous free flap & $2(4.8)$ \\
Dorsalis pedis free flap & $1(2.4)$ \\
Jejunal free flap & $1(2.4)$ \\
Total & $42(100)$ \\
\hline
\end{tabular}

Table 4. Incidence of complications associated with risk factor

\begin{tabular}{|lcc|}
\hline Risk factor & With complications & Total \\
\hline Hypertension & $1(16.7)$ & 6 \\
Diabetic mellitus & $1(33.3)$ & 3 \\
Smoking & $8(26.7)$ & 30 \\
Preoperative radiotherapy & $0(0)$ & 2 \\
Obesity & $2(29)$ & 7 \\
\hline Values are presented as number (\%). & \\
\hline
\end{tabular}

complications occurred in 4 patients (9.5\%), and minor complications occurred in 6 patients (14\%). In patients who developed major complications, one patient had venous thrombosis, and three patients had partial flap loss. In patients who developed minor complications, four patients had flap dehiscence, one patient had leakage, and one patient had difficulty in swallowing. Complication rates for patients with hypertension and diabetes were $16.7 \%$ and $33 \%$, respectively. In the smoking patient group, complications occurred in eight patients (26.7\%). There was no occurrence of complication in patients treated with preoperative radiation. In the obese patient group, complications occurred in two patients (29\%) (Table 4). In our study, according to the analysis using Fisher's exact test, the risk factors of patients did not increase the complications of free flap transfer (Table 5). In the RFFF group, two patients (8.3\%) developed partial necrosis and were managed with secondary intention healing. In the ALTPF group, two patients (25\%) developed flap dehiscence and were managed with secondary intention healing. A comparison revealed that the complication rate was higher in the ALTPF group than in the RFFF group, but without statistical significance $(25 \%$ vs. $8.3 \%, \mathrm{P}>0.05)$ (Table 5 ).

\section{DISCUSSION}

A total of 42 free flap transfers for reconstruction of surgical defects of the head and neck were considered in our study. Despite the small size of our study sample, free flap failure did not occur. All of the surgical procedures were performed by a single senior surgeon over a long period; therefore, our surgery outcomes
Table 5. Complication rate associated with risk factors and flap type

\begin{tabular}{|c|c|c|c|}
\hline & Complication & Non-complication & P-value ${ }^{a)}$ \\
\hline HTN & $1(16.7)$ & $5(83.3)$ & 1.000 \\
\hline Non-HTN & $8(22.0)$ & $28(78.0)$ & \\
\hline DM & $1(33.3)$ & $2(66.7)$ & 1.000 \\
\hline Non-DM & $5(12.8)$ & 34 (87.2) & \\
\hline Smoking & $8(26.7)$ & $22(73.3)$ & 0.696 \\
\hline Non-smoking & $2(16.7)$ & $10(83.3)$ & \\
\hline Preoperative RT & $0(0)$ & $2(100)$ & 1.000 \\
\hline Non-preoperative RT & $11(27.5)$ & $29(72.5)$ & \\
\hline Obesity & $2(29)$ & $5(71.0)$ & 1.000 \\
\hline Non-obesity & $8(27.6)$ & 21 (72.4) & \\
\hline RFFF & $2(8.3)$ & $22(91.7)$ & 0.148 \\
\hline ALTPF & $2(25)$ & $6(75)$ & \\
\hline \multicolumn{4}{|c|}{$\begin{array}{l}\text { Values are presented as number (\%). } \\
\text { HTN, hypertension; DM, diabetic mellitus; RT, radiation therapy; RFFF, radial forearm } \\
\text { free flap; ALTPF, anterolateral thigh perforator flap. } \\
\text { a'Fisher's exact test. }\end{array}$} \\
\hline
\end{tabular}

showed consistency by avoiding the influence of differences in the technical skill of multiple centers and/or multiple surgeons. In addition, to avoid inadvertent anteroposterior wall suture in microvascular anastomosis, the surgeon performed vascular anastomosis using a modified Harashina procedure and achieved leak-proof and reliable anastomosis [6].

In our study, an analysis using Fisher's exact test revealed that the risk factors of patients did not increase the incidence of complications. Similar to our report, multiple centers have previously reported that risk factors of microvascular surgery did not increase the rate of complications. Bozikov and Arnez [7] reported that only diabetic patients had a higher incidence of free flap complication, although this fact did not achieve significance in the statistical analysis. Bianchi et al. [8] reported a higher frequency of complications in patients older than 70 years, diabetic patients, and patients treated with preoperative radiation, although the differences were not statistically significant. Choi et al. [9] reported obesity as the only independent factor for the development of complications, while the number of risk factors of the patient showed an association with increased risk. In our study, two patients received treatment with preoperative radiation therapy. Among them, there was no occurrence of complications. However, although irradiated tissues were known to be risk factors for wound healing and microvascular surgery, the influence of irradiated tissue on free flap transfer has been controversial [10]. Mulholland et al. [11] and Singh et al. [12] reported that prior radiation therapy did not have a negative impact on microvascular surgery. Chao et al. [13] reported that perioperative recipient-site complications occurred at a rate similar to the rate experienced by non-irradiated patients and free flap losses were not increased despite potential damage to recipient vessels 
with neoadjuvant radiation therapy.

In our study, RFFF and ALTPF flaps were employed most often. In the RFFF group, complications occurred in $8.3 \%$ of the patients, whereas in the ALTPF group, complications occurred in $25 \%$ of the patients. However, an analysis using Fisher's exact test revealed that a larger number of complications occurred in the ALTPF group than in the RFFF group, although the difference was not statistically significant (Table 5). A review of the literature showed that our results are comparable with the results reported by others: Baek et al. [14] reported that complications occurred in $6.7 \%$ of the patients in the ALTPF group that they considered, and Smith et al. [15] reported the occurrence of complications in $18.6 \%$ of the patients in the RFFF group of their study. Similar to our results, Baek et al. [14] and Smith et al. [15] reported that most complications were venous thrombosis and congestion. Bianchi et al. [16] and Kesting et al. [17] reported several advantages of the anterolateral thigh flap for head and neck defects, including versatility, short harvesting time, and donor site morbidity. However, Kesting et al. [17] reported that intra-operative arterial spasms occurred more often in the case of the ALT free flap than in the case of RFFF and that venous congestion was the most frequent complication in the case of the RFFF. The larger diameter of veins accompanying the descending branch may be a possible reason for fewer venous complications and higher salvage rates in the case of the ALT flap than in the case of RFFF [17]. Bianchi et al. [16] reported that the ALT flap is a good donor but is not suitable for small defect areas or in cases that require a pliable or thin flap. In addition, they reported several disadvantages: in obese patients, the flap could be bulky, and in a hairy patient, hair could grow in unwanted areas. RFFF has the advantage of short operative time, but has several drawbacks, such as a visible scar on the forearm and a sacrifice of a major vessel of the hand, which could be a burden for both the surgeon and the patient. ALTPF for most head and neck reconstructions, particularly in the intraoral cavity, hypopharynx, and oropharynx, has been performed by a senior surgeon since 2007 . Nevertheless, as ALTPF is similar to RFFF with respect to the characteristics of thinness and pliability, ALTPF is a little more difficult for a novice surgeon to perform because of a longer operative time and the requirement of surgical skill with a steep competency learning curve. However, vessel anatomy has already been investigated in several previous cadaver and radiological studies, and a preoperative perforator could be identified using computed tomographic angiography. These useful tools could be helpful to young surgeons while performing ALTPF. Therefore, ALTPF is a good alternative to RFFF in patients in whom RFFF is contraindicated or who do not want to undergo RFFF.
Our study has several limitations. First, the sample size of our study was small compared to that of the other studies on this topic. Therefore, we used Fisher's exact test for the identification of factors associated with free flap complications. Second, we could not evaluate the outcomes in terms of functional aspects and patients satisfaction. Meanwhile, our study has several advantages: First, all surgical procedures were performed by a single surgeon over a long period of time; therefore, our surgical outcomes showed consistency by avoiding the influence of multiple centers and/or multiple surgeons. Second, we compared the complication rates of RFFF and ALTPF. A recent study focused mainly on the versatility and outcomes of ALT flap surgery for the reconstruction of the head and neck. However, we evaluated only ALTPF versus RFFF, which is a thin and pliable flap.

In our study, risk factors did not influence the outcomes of free flap transfer, which was in agreement with previous studies. From the results of our study, we were able to conclude that the surgeon's expertise in performing microvascular surgical techniques is an important factor for the achievement of a good result of free flap transfer. Therefore, the patient's risk factors no longer be considered a negative factor for a free flap transfer. In addition, ALTPF is a good alternative to RFFF in patients in whom it is contraindicated or who do not want to undergo RFFF.

\section{REFERENCES}

1. Cheng MH, Huang JJ. Oral cavity, tongue, and mandibular reconstructions. In: Neligan PC, editor. Plastic surgery. 3rd ed. London: Elsevier Saunder; 2013. p.307-35.

2. Lenert JJ, Evans GR. Oral cavity reconstruction In: Mathes SJ, Hentz VR, editors. Plastic surgery: Vol. III head and neck. 2nd ed. Philadelphia, PA: Saunders Elsevier Cop.; p.917-56.

3. Hurvitz KA, Kobayashi M, Evans GR. Current options in head and neck reconstruction. Plast Reconstr Surg 2006; 118:122e-33e.

4. Hsing CY, Wong YK, Wang CP, et al. Comparison between free flap and pectoralis major pedicled flap for reconstruction in oral cavity cancer patients: a quality of life analysis. Oral Oncol 2011;47:522-7.

5. Chen CH, Lin GT, Fu YC, et al. Comparison of deltopectoralis flap and free radial forearm flap in reconstruction after oral cancer ablation. Oral Oncol 2005;41:602-6.

6. Foucher G, Schuind F. A new trick for end-to-end anastomosis in microvascular surgery. Modified Harashina procedure. J Reconstr Microsurg 1984;1:49-51.

7. Bozikov K, Arnez ZM. Factors predicting free flap complications in head and neck reconstruction. J Plast Reconstr Aesthet Surg 2006;59:737-42. 
8. Bianchi B, Copelli C, Ferrari S, et al. Free flaps: outcomes and complications in head and neck reconstructions. J Craniomaxillofac Surg 2009;37:438-42.

9. Choi JW, Lew DH, Lee WJ, et al. Analysis of 180 head and neck reconstructional cases with free flap transfer. J Korean Soc Plast Reconstr Surg 2003;30:573-8.

10. Bourget A, Chang JT, Wu DB, et al. Free flap reconstruction in the head and neck region following radiotherapy: a cohort study identifying negative outcome predictors. Plast Reconstr Surg 2011;127:1901-8.

11. Mulholland S, Boyd JB, McCabe S, et al. Recipient vessels in head and neck microsurgery: radiation effect and vessel access. Plast Reconstr Surg 1993;92:628-32.

12. Singh B, Cordeiro PG, Santamaria E, et al. Factors associated with complications in microvascular reconstruction of head and neck defects. Plast Reconstr Surg 1999;103:403-11.

13. Chao AH, Chang DW, Shuaib SW, et al. The effect of neoadjuvant versus adjuvant irradiation on microvascular free flap reconstruction in sarcoma patients. Plast Reconstr Surg 2012;129:675-82.

14. Baek JH, Choi JW, Lee Wj, et al. The versatility of the anterolateral thigh perforator flap in oral cavity reconstruction after cancer ablation surgery. J Korean Soc Plast Reconstr Surg 2004;31:643-8.

15. Smith GI, O’Brien CJ, Choy ET, et al. Clinical outcome and technical aspects of 263 radial forearm free flaps used in reconstruction of the oral cavity. Br J Oral Maxillofac Surg 2005;43:199-204.

16. Bianchi B, Ferri A, Ferrari S, et al. The free anterolateral thigh musculocutaneous flap for head and neck reconstruction: one surgeon's experience in 92 cases. Microsurgery 2012; 32:87-95.

17. Kesting MR, Holzle F, Wales C, et al. Microsurgical reconstruction of the oral cavity with free flaps from the anterolateral thigh and the radial forearm: a comparison of perioperative data from 161 cases. Ann Surg Oncol 2011;18:1988-94. 